\title{
La enseñanza de la Geometría en Educación Secundaria superior
}

\section{Teaching Geometry in High Secondary Education}

\author{
Patricia Vedovatti \\ Máster en Educación, Universidad ORT Uruguay. \\ Profesora de Educación Media en Especialidad Matemática, Centro Regional de Profesores del Litoral \\ Norte, Salto, Uruguay. Docente Efectiva por concurso en el Consejo de Educación Secundaria y en el \\ Consejo de Educación Técnico - Profesional.
}

Fecha de recibido: 13/02/2014

Fecha de aceptación: 11/06/2014

\section{Resumen}

El propósito de este artículo es presentar un estudio sobre el abordaje metodológico que docentes de Bachillerato ${ }^{1}$ realizan acerca de sus prácticas de enseñanza de la Geometría, investigación a través de la cual se buscó caracterizar dichas prácticas y se atendieron las valoraciones que los docentes hacen al momento de decidir emplear o no recursos didácticos en sus aulas. Se procuró indagar en sus posturas respecto al tratamiento de las demostraciones geométricas.

Para cumplir con tales fines se trabajó con una metodología mixta, por lo que las técnicas de recolección de datos empleadas correspondieron a métodos de corte tanto cualitativo como cuantitativo.

A partir del análisis de la información recabada fue posible caracterizar las prácticas de enseñanza de la Geometría en Bachillerato como prácticas de representación material, prácticas de transformación y práctica invisible.

En primer lugar sería posible establecer que el empleo de recursos didácticos se encuentra presente en las aulas de Segundo Ciclo. Éstos servirían como soporte para el desarrollo de habilidades como la abstracción, la visualización, la argumentación, entre otras. Se destaca una fuerte tendencia al cambio en cuanto a las metodologías de abordaje de las demostraciones y las argumentaciones. Los docentes sujetos de esta investigación consideran necesaria la selección de actividades que impliquen para los estudiantes un verdadero proceso de producción Matemática.

Se observó asimismo la planificación anual como una práctica cuyos verdaderos objetivos se dejan entrever entre los discursos de este grupo de docentes, pero que en raras ocasiones son presentados por escrito.

PALABRAS CLAVE: enseñanza, aprendizaje, geometría, demostración, materiales didácticos, investigación. 


\section{Abstract}

The purpose of this paper is to present a study on the methodological approach of Higher Secondary Education teachers as regards their Geometry teaching practices, having this study the purposes of identifying such practices and assessments that teachers carry out when deciding to use or not teaching resources in their classrooms. It also sought to find out their stance regarding the treatment of geometrical demonstrations.

To meet such end, the methodology used was eclectic, so the data collection techniques employed corresponded to methods of qualitative and quantitative nature.

From the analysis of the information gathered, it was possible to characterize teaching practices of Geometry in high school as material representation practices, processing practices and invisible practices.

First of all, it would be possible to state that the use of teaching resources is present in the classrooms of high schools. This serves as a support for the development of skills such as abstraction, visualization, argumentation, among others.

There is a strong trend towards change in terms of methodologies which approach the demonstration and argumentation. The teachers who are the subjects of this research, considered necessary to select activities that involve students in real Math production.

Apart from this, the annual plan was also observed as a type of practice whose real objectives are partly seen as speeches of this group of teachers and it is hardly ever presented in written form.

KEYWORDS: Teaching, Learning, Geometry, Demonstration, Teaching Materials Research.

\section{Introducción}

El presente artículo es el resultado de una investigación llevada a cabo durante los años 2012 - 2013 en liceos públicos de una ciudad del interior del país.

En todas las ramas de la educación existen innumerables factores que afectan las maneras en que son llevadas a cabo las prácticas de enseñanza. En esta oportunidad, se intentó poner de manifiesto la preocupación existente por las metodologías de abordaje de la Geometría en Bachillerato.

Si bien en los distintos cursos de educación formal se busca que el estudiante dé prueba de la credibilidad de una afirmación, desde hace varios años autores como Godino y Recio (2001), Martín y Harel (1989; en Godino y Recio, 2001), Martínez Recio (2001), ANEP (2007) discuten sobre las dificultades que implica la enseñanza de las demostraciones, particularmente en lo que a la Geometría refiere.

En el trabajo realizado -en el marco de los estudios de la Maestría en Educación de la Universidad ORT- se procuró atender alguna de estas inquietudes, con el objetivo de caracterizar aquellas cuestiones tenidas en cuenta por los docentes de Bachillerato al momento de seleccionar las metodologías de enseñanza de la Geometría. A tal fin se buscó dar respuesta a dos interrogantes que resultaron básicas para la investigación llevada a cabo: ¿Cuáles son las estrategias de enseñanza mayormente empleadas por los docentes de Bachillerato, en particular las valoraciones que éstos hacen acerca del empleo de recursos didácticos para la enseñanza de la Geometría? y ¿cuál es su postura con respecto al abordaje de las prácticas argumentativas y el tratamiento de las demostraciones geométricas?

\section{Algunas consideraciones teóricas}

En este trabajo de investigación se entendió que la Geometría se caracteriza por presentar una gran adaptabilidad ante el diseño de diversas estrategias. Por tal motivo, se compartió con Itzcovich (2005) la premisa de que la práctica de la Geometría acerca al estudiante a vivir la cultura de una forma diferente ya que la propia experiencia del pensar geométricamente 
presenta características de dominio diferentes a las de otras áreas. Pensar geométricamente, según Azcárate (2003), es razonar sobre un objeto geométrico poniendo en juego procesos de pensamiento tales como el representar, visualizar, interpretar, clasificar, abstraer, conjeturar, analizar, probar hipótesis y generalizar. Cabe destacar que el pensamiento geométrico involucra un conocimiento matemático más avanzado, donde quien aprende ha de entrar en contacto con el objeto geométrico, "objetos... (puntos, figuras, cuerpos, etc.) que no pertenecen a un espacio físico real sino a un espacio teórico, conceptualizado" (Itzcovich, 2005; 10).

Se asumió que a medida que el estudiante atraviesa por los distintos niveles de Educación Secundaria comienza a enfrentarse a cursos que implican cada vez mayor grado de abstracción conceptual. Díaz Godino, Gómez, Gutiérrez y Rico (1999) plantean que para que se dé una situación de aprendizaje se torna necesario que quien enseña habilite espacios que provoquen la interacción entre el alumno, los sentidos (en interacción con el entorno) y el deseo de saber. La comprensión de las formas y relaciones de los objetos provenientes del entorno real permite el desarrollo de las operaciones cognitivas necesarias para el reconocimiento de formas y propiedades geométricas. Representar el concepto geométrico en un espacio físico o virtual supondría implicar un fuerte pie de apoyo para iniciar al estudiante en el estudio geométrico. Pese a esto, el pensamiento geométrico no puede quedar ligado al espacio material. El alumno ha de "tomar en cuenta el objeto de conocimiento" (Brousseau, 2007; 98), ha de avanzar en sus formas de razonamiento y lograr así establecer las representaciones mentales de los objetos en cuestión.

Se propuso, asimismo, que el trabajo de aula a partir de la modelización matemática ${ }^{2}$, la resolución de problemas y la interacción con otros coloca al estudiante en una situación de producción de conocimiento donde el explorar, interpretar y organizar establece un acercamiento al objeto matemático y por tanto a la construcción de los saberes.

Sin embargo, tanto el nivel de complejidad de los contenidos a trabajar como las exigencias del curso a los que deberá hacer frente el estudiante aumentan notoriamente conforme el pasaje de grado.

Según Godino y Recio (2001) los programas de los diferentes cursos de educación formal establecen que, ya sea por verificación o experimentación en los primeros años o por la elaboración de rigurosos métodos deductivos en los niveles más avanzados, los estudiantes han de dar argumentos que den cuenta de la garantía de sus producciones.

Para lograr avanzar en forma paulatina en el proceso de aprendizaje de la argumentación, de la Torre establece que "La prueba, debería ser parte del proceso de resolución de problemas, logrando que los estudiantes fuesen capaces de mezclar deducción y experimentación, idear representaciones, trazar diagramas, moverse entre diferentes representaciones" (de la Torre, 2003; 2).

Se consideró de relevancia aceptar, además, que en el enseñar es ineludible tener en cuenta tanto la metodología a emplear para el tratamiento de un concepto como la problemática del sujeto que aprende. Desde esta perspectiva se adhiere al planteo establecido por Edelstein quien sostiene que "La construcción metodológica... se conforma a partir de la estructura conceptual... de la disciplina y la estructura cognitiva de los sujetos en situación de apropiarse de ella" (Edelstein, 1996, en Camilloni et al., 1996; 81:82). 


\section{En síntesis}

A lo largo de este estudio se buscó caracterizar las prácticas de enseñanza de la Geometría impartidas por un grupo de docentes de Bachillerato. Para ello se consideró necesario atender a la forma en la que quienes participaron de esta investigación seleccionan las estrategias de enseñanza en función de los contenidos a abordar, los alumnos con los que trabajan y el contexto en el cual se encuentran inmersos.

\section{Metodología}

Para estudiar el abordaje de la metodología de enseñanza de la Geometría que hacen los docentes de Bachillerato fue necesario definir los caminos a seguir para dar respuesta a las interrogantes planteadas.

Este trabajo fue abordado desde un paradigma tanto cuantitativo como cualitativo, entendiendo que la integración de ambos modelos brindaría mayor riqueza interpretativa y favorecería la construcción de conocimientos.

El empleo de un modelo mixto permitió desarrollar conocimiento, construir teoría y resolver problemas (Hernández Sampieri et al., 2010).

La producción de conocimiento sobre las prácticas de enseñanza de la Geometría en Bachillerato a través de un enfoque cuantitativo se llevó a cabo mediante la aplicación de una encuesta a docentes. Con ello fue posible obtener una visión general de las principales cuestiones tenidas en cuenta al momento de seleccionar las metodologías de abordaje de la Geometría. La encuesta permitió contextualizar la realidad de los profesores sujetos de esta investigación ya que con ella fue posible recabar datos que dieran cuenta de las características más sobresalientes de la población con la que se estaba trabajando.

El empleo de un modelo cualitativo permitió un acercamiento fenomenológico al objeto de estudio. Mediante entrevistas a docentes y a un experto en el área fue posible recabar y contrastar experiencias, percepciones y creencias, lo que permitió comprender cómo estos docentes definen y construyen sus prácticas de enseñanza de la Geometría.

\section{El diseño}

En esta fase del trabajo de investigación fue necesaria la determinación de las particularidades metodológicas a implementar para llevar a cabo el proceso de siembra e interpretación. "Diseñar", expone Valles, "significa, ante todo, tomar decisiones a lo largo de todo el proceso de investigación y sobre todas las fases o pasos que conlleva dicho proceso" (Valles, 2007; 78).

Pensar en el diseño de investigación implicó planificar estrategias para la recolección y el análisis de datos que permitieran dar respuesta a las interrogantes planteadas.

El trabajo de campo se llevó a cabo en cuatro momentos y requirió del empleo de estrategias de recolección de datos en forma correlacional, proponiéndose un acoplamiento de la entrevista a la encuesta y estableciéndose en forma casi paralela el estudio de documentos. La aplicación de cada técnica abrió camino a la siguiente modalidad de recolección de datos lo que permitió beneficiarse de los puntos fundamentales de cada una de ellas y cruzar los resultados obtenidos (Rodríguez Ruiz, 2005). 
El siguiente cuadro da cuenta de los momentos transcurridos durante el proceso de obtención y análisis de datos:

\begin{tabular}{|l|l|}
\hline \multirow{2}{*}{$\begin{array}{c}\text { Momentos del } \\
\text { trabajo de campo }\end{array}$} & 1. Estudio de planes y programas propuestos por el CES. \\
\cline { 2 - 2 } & 3. Encuestas a docentes. \\
\cline { 2 - 2 } & 4. Estudio de la documentación elaborada por cada docente. \\
\hline
\end{tabular}

\section{Sobre el material documental}

La primera estrategia metodológica empleada en este trabajo correspondió a la búsqueda, selección y análisis de planes y programas vigentes. Se estudió todo tipo de archivos que permitieran distinguir estrategias de enseñanza aplicadas por los docentes de Bachillerato al momento de enfrentarse a sus clases de Geometría.

La exploración, clasificación y revisión de este tipo de fuentes secundarias permitió seleccionar la población a trabajar: docentes de Bachillerato del plan 2006 cuyos programas integraran el tratamiento de contenidos geométricos.

Una segunda instancia de análisis del material documental se originó a partir del estudio de los planes anuales confeccionados por algunos de los docentes que participaron del trabajo de campo (quienes posteriormente participaron del proceso de entrevistas).

Finalizada esta fase se efectuó un análisis comparativo entre programas de Bachillerato propuestos por el CES (Consejo de Educación Secundaria) y planes anuales elaborados por los docentes.

Las conclusiones obtenidas fueron consideradas durante el proceso de triangulación realizado posteriormente.

\section{Encuestas a docentes}

Mediante la aplicación de encuestas se buscó capturar la realidad de la población. Un cuestionario auto-administrado permitió recabar información tanto de hechos como de opiniones de los profesores de Bachillerato al momento de pensar sus clases de Geometría (secuencia de clase, empleo de recursos informáticos, materiales concretos o tradicionales, trabajo en grupos o en forma individual, actividades que impliquen la resolución de problemas, entre otros). En especial se estudiaron las valoraciones que éstos le asignan al trabajo con recursos didácticos, en particular al momento de seleccionar las estrategias de enseñanza de las argumentaciones y demostraciones geométricas.

La aplicación de las encuestas se llevó a cabo en aquellos liceos públicos de una ciudad del interior del país que contaban con cursos completos de Bachillerato plan 2006. Finalizado el procesamiento y análisis de la información recogida mediante las encuestas se procedió a la aplicación de entrevistas a un grupo de docentes que había participado de esta instancia. De este modo fue posible complementar los resultados obtenidos hasta el momento ya que la aplicación de entrevistas favoreció la comprensión de algunas de las respuestas proporcionadas en la mencionada encuesta. 


\section{De las entrevistas}

En primer lugar, se efectuaron entrevistas basadas en un guión (Patton, 1990 en Valles, 2007) a docentes, lo que permitió un acercamiento fenomenológico al objeto estudiado. Quienes participaron de esta instancia describieron percepciones, experiencias, metodologías de trabajo y creencias acerca de las prácticas de enseñanza de la Geometría, en particular sobre el abordaje de las demostraciones geométricas y el tratamiento de las argumentaciones en el aula.

Culminada esta instancia se procedió a la aplicación de una entrevista a un actor clave. Ésta fue constituida en base a los datos recabados durante las entrevistas a docentes. Una vez más el empleo de una entrevista basada en un guión permitió sostener una conversación con un experto en el área, centrada en sus experiencias personales y la libertad de expresión de sus opiniones.

Mediante una serie de preguntas y de fragmentos recortados de las expresiones brindadas por los propios profesores se hizo posible la contrastación de opiniones y argumentos.

\section{Hallazgos}

Posicionarse como investigador frente al objeto de estudio, recabar información, estudiarla y contrastarla, no resultó tarea simple. Tratar de indagar sobre las prácticas de enseñanza de la Geometría en cursos de Bachillerato implicó, una vez más, recordar que no existen recetas o patrones que oficien de guía del cómo trabajar en el aula.

Sin embargo, en la investigación desarrollada se detectaron puntos de encuentro entre las metodologías de enseñanza llevadas a cabo por los docentes sujetos de esta investigación. De allí es que fue posible categorizar las prácticas de enseñanza de la Geometría en Bachillerato desde dos dimensiones: una enfocada directamente a la enseñanza de la Geometría y la otra desde una perspectiva que abarca la planificación.

\begin{tabular}{|c|c|c|}
\hline \multirow{2}{*}{$\begin{array}{c}\text { Prácticas de } \\
\text { enseñanza de } \\
\text { la Geometría en } \\
\text { Bachillerato }\end{array}$} & Dimensión 1 & a. Prácticas de representación material \\
\cline { 3 - 3 } & & b. Práctica de transformación \\
\cline { 2 - 3 } & Dimensión 2 & C. Práctica invisible \\
\hline
\end{tabular}

\section{Prácticas de representación material}

Las prácticas de representación material atienden al empleo de recursos didácticos en el aula como soporte para el desarrollo de habilidades tales como la visualización, la abstracción, la elaboración de conjeturas y la argumentación, entre otras. Este tipo de prácticas forma parte de un cambio que se viene presentando en el Bachillerato en cuanto a las formas de abordaje de los contenidos geométricos.

Por un lado, se busca favorecer los procesos de aprendizaje. Se presenta un fuerte interés por lograr una correcta selección y organización de actividades de modo que los contenidos geométricos sean presentados en forma secuencial y escalonada.

Por el otro, existe una enorme preocupación por los procesos de aprendizaje de los estudiantes. Los docentes que participaron de la investigación entienden que la actividad intelectual y de reflexión que los estudiantes realicen durante el proceso de aprehensión de los conocimientos favorece el tránsito por los diferentes procesos de pensamiento -el pasaje de lo concreto a lo abstracto-.

Se seleccionan estrategias que integren trabajos grupales, se promueve la discusión y argumentación de ideas y se entiende que representar materialmente el objeto geométrico con el que se está trabajando es el medio para lograrlo. 
Los profesores de Bachillerato sujetos de esta investigación buscan proponer actividades que impliquen el empleo de recursos didácticos, particularmente software educativo con la finalidad de representar las situaciones propuestas. El objeto geométrico, un objeto perteneciente a un espacio construido desde una teoría, es representado materialmente a partir de un recurso didáctico. Estos actuarían a modo de sustento para la actividad intelectual que el estudiante ha de realizar para la construcción de los conceptos.

En la representación material a través de un recurso didáctico (materiales concretos, materiales tradicionales, software educativo, entre otros) el objeto geométrico que se desea abordar es presentado por docentes de los cursos más avanzados de Educación Secundaria como soporte de inicio para el desarrollo de habilidades como la visualización, la abstracción, la elaboración de conjeturas y la argumentación.

\section{Prácticas de transformación}

Estas prácticas forman parte de acciones realizadas con el fin de transformar las metodologías de abordaje de las demostraciones y las prácticas argumentativas.

Se busca romper con el modelo educativo tradicional que hasta el momento parecería continuar presente en las aulas de Bachillerato. El rol protagónico del profesor sufre un quiebre comenzando a cobrar fuerza la voz del estudiante.

Los docentes buscan desvincular los lazos que los unen a sus experiencias como estudiantes, para lo que presentan modos alternativos para el tratamiento de las demostraciones. Trabajo en grupos, resolución de problemas, trabajo con fichas, empleo de recursos didácticos, entre otros, son algunas de las estrategias propuestas en el aula para que los estudiantes aprendan el funcionamiento del razonamiento deductivo.

Comienzan a implementarse actividades de enseñanza que obliguen a los estudiantes a pensar y reflexionar sobre la propuesta tomando en consideración sus conocimientos previos. Se plantean situaciones que impliquen la discusión e intercambio de ideas y permitan al alumno arribar a las conclusiones deseadas mediante la elaboración y contrastación de conjeturas.

\section{Práctica invisible}

En el estudio realizado se halló que una de las tareas que parecería ser de menor agrado para los docentes es la de registrar en forma escrita un plan de intencionalidades a seguir a lo largo del año -metodologías de trabajo, recursos a implementar, estrategias de enseñanzacon el fin de cumplir con los objetivos propuestos en cada curso.

De cierto modo estos docentes parecían no interesarse en la construcción de un plan anual que diera cuenta del proceso reflexivo realizado al momento de plasmar qué hacer en el aula y cómo llevar a cabo tal labor. Sin embargo, se halló que este aspecto se presentaba bajo una apariencia un tanto distorsionada de la realidad. A pesar de la ausencia de registro de dichas intencionalidades, el proceso de reflexión, autonomía pedagógica y libertad en cuanto a la toma de decisiones del accionar en el aula era una práctica realizada en forma habitual por este grupo de docentes.

Trabajar en forma coordinada, discutir propuestas de enseñanza, fundamentar contenidos a priorizar, intercambiar experiencias de aula, analizar y seleccionar estrategias metodológicas, entre otras, son prácticas habituales para los docentes de Bachillerato al momento de enfrentarse al tratamiento de contenidos geométricos. Pero por algún motivo el plan anual no siempre es visto como el espacio donde plasmar estas acciones de trabajo, por lo que este proceso reflexivo parecería constituirse en una práctica invisible ante los ojos de un observador ajeno a esta realidad. 


\section{Consideraciones finales}

Los datos recogidos y analizados durante esta investigación permiten concluir que en el ámbito educativo se detecta una tendencia al cambio en cuanto a la metodología de abordaje de las prácticas de enseñanza de la Geometría en los niveles superiores de Educación Media. La caracterización presentada en este estudio sobre las prácticas de enseñanza permitió rescatar una Geometría que parecía estar olvidada y sin brillo e hizo posible acercar una visión positiva de las formas de tratamiento de los contenidos geométricos en Bachillerato.

Sin embargo, finalizando el trabajo se presentaron nuevas inquietudes en las cuales se debía ahondar. Muchas de las cuestiones de las cuales se valían los docentes sujetos de esta investigación para la enseñanza de los contenidos geométricos mostraron puntos de encuentro con las estrategias y metodologías de enseñanza usualmente empleadas en Ciclo Básico. En este orden de cosas, ¿podría decirse que existe una "ciclobasicalización" del Bachillerato? En este sentido se estaría introduciendo el término "ciclobasicalización” para hacer referencia a si las metodologías de abordaje de la Geometría en Ciclo Básico y en Bachillerato son las mismas. En tal caso, las estrategias de enseñanza empleadas en ambos ciclos educativos ¿son las mismas? Y de ser así, ¿cómo se implementa su uso?

\section{Bibliografía}

Administración Nacional de Educación Pública (ANEP), Consejo de Educación Secundaria (CES). (2010) Planes y programas: Ajustes 2010 Programas de Matemática de Bachillerato. Reformulación 2006. Accedido el 10/07/2012, desde http://www.ces.edu.uy/ces/images/stories/ reformulacion06/ajustesprogmat2010/ajustes2010progmatdivcientifica5ref2006.pdf

Administración Nacional de Educación Pública (ANEP). Dirección Sectorial de Planificación Educativa Dirección de Investigación, Evaluación y Estadística (2007). La enseñanza y los aprendizajes de matemática en el primer ciclo de educación media. Montevideo: Tradinco S.A.

Administración Nacional de Educación Pública (ANEP) (2011). La evaluación de la Competencia Matemática PISA 2012. Accedido el 02/04/2013, desde http://www.anep.edu.uy/anep/ phocadownload/pisa/pisa2012/Informestematicos/Matematica\%20en\%20PISA\%202012.pdf

Azcárate, C. (2003). Definiciones, demostraciones, ¿por qué?, ¿cuándo?, ¿cómo? En: Ponencia en las X JAEM. Accedido el 10/02/2012, desde http://www.quadernsdigitals.net/ datos/hemeroteca/r_40/nr_456/a_6218/6218.pdf

Brousseau, G. (2007). Iniciación al estudio de la teoría de las situaciones didácticas. Buenos Aires: Libros del Zorzal.

Camilloni, A.; Davini, C.; Edelstein, G.; Litwin, E.; Souto, M. \& Barco, S. (1996). Corrientes didácticas contemporáneas. Buenos Aires: Paidós.

Díaz Godino, J.; Gómez, B.; Gutiérrez, Á. \& Rico, L. (1999). Área de conocimiento: Didáctica de la Matemática. Madrid: Síntesis.

Godino, J. \& Recio, Á. (2001). Significados institucionales de la demostración. Implicaciones para la educación Matemática. Accedido el 03/02/12, desde http://ddd.uab.es/pub/ edlc/02124521v19n3p405.pdf

Hernández Sampieri, R.; Fernández Collado, C.; Baptista Lucio, P. (2010). Metodología de la Investigación Social. 5ta Ed. México: Mac Graw Hill.

Itzcovich, H. (2005). Iniciación al estudio didáctico de la Geometría. Buenos Aires: Libros del Zorzal. 
Martínez Recio, A. (2001). La demostración en matemática. Una aproximación epistemológica y didáctica. Universidad de Córdoba. Accedido el 04/04/2012, desde http://scholar.google.com/ scholar?q=demostraci\%C3\%B3n+en+matematicaa\&hl=es\&lr

Rodríguez Ruiz, O. (2005). La Triangulación como Estrategia de Investigación en Ciencias Sociales. Revista de Investigación en Gestión de la Innovación y Tecnología. LA I+D QUE TENEMOS. 31. Accedido el 01/11/12, desde http://www.madrimasd.org/revista/revista31/ tribuna/tribuna2.asp

Torre de la, E. (2003). Didáctica de la geometría y demostración de propiedades. Accedido el 18/02/2012, desde http://www.uv.es/aprengeom/archivos2/DeLaTorre03.pdf

Valles, M. (2007). Técnicas cualitativas de investigación social. Reflexión metodológica y práctica profesional. Madrid: Síntesis.

\section{Notas}

${ }^{1}$ La Educación Secundaria en el Uruguay se encuentra dividida en dos tramos denominados "ciclos educativos". El primero, Educación Básica o Ciclo Básico, con una duración de tres años, a la que asisten alumnos que han finalizado la Primaria. La finalización del tercer año de Ciclo Básico es considerada como el tramo educativo obligatorio a ser realizado por un estudiante uruguayo. El segundo ciclo, denominado Bachillerato, con la misma duración que el anterior, al que concurren estudiantes que desean culminar sus estudios secundarios y el cual habilita a continuar estudios terciarios.

${ }^{2}$ Modelización Matemática: Transformación de una situación problema presente en un contexto real en un problema matemático. Mediante el empleo de una serie de herramientas y procedimientos se llega a un resultado que se ajusta al problema formulado matemáticamente. Éstos han de ser interpretados en contexto inicial con el fin de determinar la validez o no de la solución obtenida matemáticamente dentro del contexto que dio origen al problema (PISA, 2012). 